\title{
Wiener-Itô Chaos Expansion of Hilbert Space Valued Random Variables
}

\author{
M. A. Alshanskiy \\ Ural Federal University, No. 19, Mira Street, Ekaterinburg 620002, Russia \\ Correspondence should be addressed to M. A. Alshanskiy; mxalsh@gmail.com
}

Received 23 November 2013; Accepted 13 February 2014; Published 7 April 2014

Academic Editor: Farrukh Mukhamedov

Copyright (C) 2014 M. A. Alshanskiy. This is an open access article distributed under the Creative Commons Attribution License, which permits unrestricted use, distribution, and reproduction in any medium, provided the original work is properly cited.

The notion of $n$-fold iterated Itô integral with respect to a cylindrical Hilbert space valued Wiener process is introduced and the Wiener-Itô chaos expansion is obtained for a square Bochner integrable Hilbert space valued random variable. The expansion can serve a basis for developing the Hilbert space valued analog of Malliavin calculus of variations which can then be applied to the study of stochastic differential equations in Hilbert spaces and their solutions.

\section{Introduction}

The Wiener-Itô chaos expansion of a square integrable random variable which was first proved in [1] plays fundamental role in Malliavin calculus of variations $[2,3]$ which appeared to be a powerful instrument in the analysis of functionals of Brownian motion. The Malliavin calculus has found extensive applications to stochastic differential equations arising as models of various random phenomena. One of the important sources of such equations is markets modeling in financial mathematics $[4,5]$.

In the last decades, many researchers' interest has been drawn to stochastic differential equations in infinite dimensional Hilbert spaces driven by a cylindrical Wiener process or, equivalently, by countable set of Brownian motions [6, 7]. For example, in financial mathematics, such equations are used in modeling interest rates term structure or zerocoupon bond market $[8,9]$. The present work was motivated by the need to make the Malliavin calculus applicable to Hilbert space valued stochastic processes. The first step in this direction is to obtain the generalization of the Wiener-Itô chaos expansion for Hilbert space valued random variables.

In order to achieve this, we first prove the Hilbert space valued version of the Itô representation theorem in Section 2. This generalization is established in Theorem 9 and Corollary 11.

In Section 3, we introduce iterated Itô integrals and multiple Itô integrals with respect to a cylindrical Wiener process. In the Hilbert space valued case, the integrand of an $n$-fold iterated Itô integral is a function defined on a certain subset of $\mathbb{R}^{n}$ and taking values in a certain space of Hilbert space valued continuous $n$-linear forms defined on the $n$th Cartesian power of the Hilbert space where the Wiener process takes values.

Section 4 contains main results of the paper which are stated in Theorem 9 and Corollary 11. The proof of the theorem follows the scheme of the proof of the Wiener-Itô chaos expansion in the $\mathbb{R}$-valued case in [5].

\section{Itô Representation Theorem for Hilbert Space Valued Random Variables}

Let $(\Omega, \mathscr{F}, P)$ be a probability space. For any separable Hilbert space $\mathbb{H}$, we denote by $L^{2}(\Omega, \mathscr{F}, P ; \mathbb{H})$, or $L^{2}(\Omega ; \mathbb{U})$ for short, the space of all Bochner square integrable $\mathbb{U}$-valued random variables on $(\Omega, \mathscr{F}, P)$. Let $\left\{e_{j}\right\}$ be an orthonormal basis in $\mathbb{W}$ and let $\left\{\beta_{j}(t) \mid t \in \mathbb{R}\right\}_{j=1}^{\infty}$ be a sequence of independent identically distributed Brownian motions on the probability space. Consider the corresponding cylinder Wiener process, defined by

$$
W(t)=\sum_{j=1}^{\infty} \beta_{j}(t) e_{j}, \quad t \in \mathbb{R} .
$$


It is easy to see that the series is not convergent in $L^{2}(\Omega ; \mathbb{1})$; however, for any $x \in \mathbb{R}$, we have that $(W(t), x):=$ $\sum_{j=1}^{\infty} \beta_{j}\left(e_{j}, x\right)_{\mathbb{H}}$ is a random variable belonging to $L^{2}(\Omega ; \mathbb{R})$. Denote by $\mathscr{B}_{t}^{a}, t \geq a$ the $\sigma$-algebra, generated by the Wiener process at $[a ; t]$, that is, the $\sigma$-algebra, generated by the set of random variables $(W(s), x)$, where $a \leq s \leq t, x \in \mathbb{W}$. (Note that the series $(1)$ is not convergent in $L^{2}(\Omega ; \mathbb{M})$ at $t \neq 0$ although $(W(s), x) \in L^{2}(\Omega ; \mathbb{R})$ for any $s \in \mathbb{R}, x \in$ $\mathbb{W}$.) The family $\left\{\mathscr{B}_{t}^{a}\right\}$ is called the filtration generated by the Wiener process $W(t), t \geq a$. Note that the Brownian motions $\beta_{j}(t), j \in \mathbb{N}, t \geq a$ are martingales with respect to the filtration $\mathscr{B}_{t}^{a}$.

Let $H$ be another separable Hilbert space and let $\left\{g_{i}\right\}_{i=1}^{\infty}$ be an orthonormal basis in $H$. Then, the family of operators $\left\{g_{i} \otimes e_{j}\right\}_{i, j=1}^{\infty}$, defined by the equality

$$
\left(g_{i} \otimes e_{j}\right) x:=g_{i}\left(e_{j}, x\right)_{\mathbb{W}}, \quad x \in \mathbb{U},
$$

forms an orthonormal basis in the space $\mathscr{L}_{2}(\mathbb{\boxplus}, H)$ of all Hilbert-Schmidt operators acting from $\mathbb{E}$ to $H$. Any $A \in$ $\mathscr{L}_{2}(\mathbb{\square}, H)$ has the decomposition

$$
A=\sum_{i, j=1}^{\infty} a_{i j}\left(g_{i} \otimes e_{j}\right),
$$

where $a_{i j}=\left(g_{i}, A e_{j}\right)_{H}$ and

$$
\|A\|_{\mathscr{L}_{2}(\mathbb{N}, H)}^{2}=\sum_{i, j=1}^{\infty}\left|a_{i j}\right|^{2}<\infty .
$$

For any $\mathscr{L}_{2}(\mathbb{\square} ; H)$-valued random process $\varphi(t)$ adapted to the filtration $\mathscr{B}_{t}^{a}$, where $a \leq t \leq b$, satisfying the property

$$
E\left[\int_{a}^{b}\|\varphi(t)\|_{\mathscr{L}_{2}(\uplus, H)}^{2} d t\right]<\infty,
$$

the stochastic Itô integral with respect to the cylindrical Wiener process

$$
\int_{a}^{b} \varphi(t, \omega) d W(t)
$$

is well-defined and is an element of the space $L^{2}(\Omega ; H)$ (see the definition and the properties in [6]). Note that if the function $\varphi(t)=\varphi(t, \omega)$ is $\mathscr{B}(\mathbb{R}) \times \mathscr{F}$-measurable, the equality (5) implies

$$
E\left[\int_{a}^{b}\|\varphi(t)\|_{\mathscr{L}_{2}(\boxplus, H)}^{2} d t\right]=\int_{a}^{b} E\left[\|\varphi(t)\|_{\mathscr{L}_{2}(\mathbb{H}, H)}^{2}\right] d t .
$$

Theorem 1. For any $\mathscr{B}_{b}^{a}$-measurable random variable $F \in$

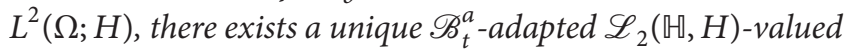
random process $\varphi(t)$ satisfying (5) such that

$$
\begin{gathered}
F=E[F]+\int_{a}^{b} \varphi(t, \omega) d W(t), \\
\|F\|_{L^{2}(\Omega ; H)}^{2}=\|E[F]\|_{H}^{2}+E\left[\int_{a}^{b}\|\varphi(t)\|_{\mathscr{L}_{2}(\mathbb{H}, H)}^{2} d t\right] .
\end{gathered}
$$

Proof. For any $i \in \mathbb{N}$, we have $F_{i}:=\left(F, g_{i}\right)_{H} \in L^{2}(\Omega ; \mathbb{R})$. Therefore, we have the following decomposition for $F$ :

$$
F=\sum_{i=1} F_{i} g_{i} .
$$

Denote $F_{\text {in }}=E\left[F_{i} \mid \mathscr{B}_{b}^{a,(n)}\right]$, where $\mathscr{B}_{b}^{a,(n)}$ is the filtration generated by the $n$-dimensional Wiener process

$$
W_{n}(t)=\sum_{j=1}^{n} \beta_{j}(t) e_{j}, \quad a \leq t \leq b .
$$

By the finite dimensional Itô representation theorem ([10], Theorem 4.3.3.), there exists a unique $\mathbb{R}^{n}$-valued random process

$$
\varphi_{\text {in }}(t)=\left[v_{i 1}^{(n)}(t), \ldots, v_{i n}^{(n)}(t)\right], \quad a \leq t \leq b,
$$

such that the following conditions hold:

(i) all the mappings $v_{i k}^{(n)}:(t, \omega) \mapsto v_{i k}^{(n)}(t, \omega)$ are $\mathscr{B}(\mathbb{R}) \times$ $\mathscr{F}$-measurable,

(ii) $E\left[\int_{a}^{b} v_{i k}^{(n)}(t)^{2} d t\right]<\infty, k=1, \ldots, n$,

(iii) the processes $v_{i k}^{(n)}(t), k=1, \ldots, n$ are adapted to the filtration $\mathscr{B}_{t}^{a}$.

Moreover, we have

$$
\begin{aligned}
F_{\text {in }} & =E\left[F_{\text {in }}\right]+\int_{a}^{b} \varphi_{\text {in }}(t) d B_{n}(t) \\
& =E\left[F_{i}\right]+\sum_{k=1}^{n} \int_{a}^{b} v_{i k}^{(n)}(t) d \beta_{k}(t) .
\end{aligned}
$$

Here, $B_{n}(t)$ denotes the $n$-dimensional Brownian motion

$$
B_{n}(t)=\left[\begin{array}{c}
\beta_{1}(t) \\
\vdots \\
\beta_{n}(t)
\end{array}\right] .
$$

Since $\mathscr{B}_{b}^{a,(n)} \subseteq \mathscr{B}_{b}^{a,(m)}$ for $m>n$, we have

$$
\begin{aligned}
F_{j n} & =E\left[F_{j} \mid \mathscr{B}_{b}^{a,(n)}\right]=E\left[E\left[F_{j} \mid \mathscr{B}_{b}^{a,(m)}\right] \mid \mathscr{B}_{b}^{a,(n)}\right] \\
& =E\left[F_{j m} \mid \mathscr{B}_{b}^{a,(n)}\right] \\
& =E\left[E\left[F_{i m}\right]+\sum_{k=1}^{m} \int_{a}^{b} v_{i k}^{(m)}(t) d \beta_{k}(t) \mid \mathscr{B}_{b}^{a,(n)}\right] \\
& =E\left[F_{i}\right]+\sum_{k=1}^{n} \int_{a}^{b} v_{i k}^{(m)}(t) d \beta_{k}(t) .
\end{aligned}
$$

It follows from the uniqueness of the representation (12) that $v_{i k}^{(m)} \equiv v_{i k}^{(n)}$ for $k \leq n<m$. Thus, the equality (12) can be rewritten as

$$
\begin{aligned}
F_{i n} & =E\left[F_{i n}\right]+\int_{a}^{b} \varphi_{i n}(t) d B_{n}(t) \\
& =E\left[F_{i}\right]+\sum_{k=1}^{n} \int_{a}^{b} v_{i k}(t) d \beta_{k}(t) .
\end{aligned}
$$


By the Jensen inequality, we have

$$
F_{i n}^{2}=E\left[F_{i} \mid \mathscr{B}_{b}^{a,(n)}\right]^{2} \leq E\left[F_{i}^{2} \mid \mathscr{B}_{b}^{a,(n)}\right] .
$$

Therefore,

$$
E\left[F_{i n}^{2}\right] \leq E\left[E\left[F_{i}^{2} \mid \mathscr{B}_{b}^{a,(n)}\right]\right]=E\left[F_{i}^{2}\right]<\infty .
$$

Thus,

$$
\begin{aligned}
E\left[F_{i n}^{2}\right] & =E\left[\left(E\left[F_{i}\right]+\sum_{k=1}^{n} \int_{a}^{b} v_{i k}(t) d \beta_{k}(t)\right)^{2}\right] \\
& =E\left[F_{i}\right]^{2}+\sum_{k=1}^{n} E\left[\int_{a}^{b} v_{i k}^{2}(t) d t\right] \leq E\left[F_{i}^{2}\right]<\infty .
\end{aligned}
$$

Consequently,

$$
\sum_{k=1}^{\infty} E\left[\int_{a}^{b} v_{i k}^{2}(t) d t\right]<\infty
$$

Since $\mathscr{B}_{b}^{a,(n)} \subseteq \mathscr{B}_{b}^{a,(n+1)}$ and $\mathscr{B}_{b}^{a}=\bigcup_{n \in \mathbb{N}} \mathscr{B}_{b}^{a,(n)}$, we have

$$
\begin{gathered}
F_{i}=\lim _{n \rightarrow \infty} E\left[F_{i} \mid \mathscr{B}_{b}^{a,(n)}\right]=E\left[F_{i}\right]+\sum_{k=1}^{\infty} \int_{a}^{b} v_{i k}(t) d \beta_{k}(t), \\
E\left[F_{i}^{2}\right]=\lim _{n \rightarrow \infty} E\left[F_{i n}^{2}\right]=E\left[F_{i}\right]^{2}+\sum_{k=1}^{\infty} E\left[\int_{a}^{b} v_{i k}^{2}(t) d t\right] .
\end{gathered}
$$

For any $N \in \mathbb{N}$, it holds that

$$
E\left[\sum_{i=1}^{N} F_{i}^{2}\right]=\sum_{i=1}^{N} E\left[F_{i}\right]^{2}+\sum_{i=1}^{N} \sum_{k=1}^{\infty} E\left[\int_{a}^{b} v_{i k}^{2}(t) d t\right] \leq E\left[\|F\|_{H}^{2}\right] .
$$

It follows from (20) that

$$
\sum_{i=1}^{\infty} F_{i} g_{i}=\sum_{i=1}^{\infty} E\left[F_{i}\right] g_{i}+\sum_{i=1}^{\infty} g_{i} \sum_{k=1}^{\infty} \int_{a}^{b} v_{i k}(t) d \beta_{k}(t),
$$

since the series in the right-hand side of the equality are convergent in $L^{2}(\Omega ; H)$ by the estimate (21). Thus, the equality (8) holds true. It also follows from this estimate by the Levy theorem that we can pass to the limits at $N \rightarrow \infty$ in the equality. As a result, we obtain

$$
\begin{aligned}
E\left[\|F\|_{H}^{2}\right]= & \sum_{i=1}^{\infty} E\left[F_{i}\right]^{2} \\
& +\sum_{i=1}^{\infty} \sum_{k=1}^{\infty} E\left[\int_{a}^{b} v_{i k}^{2}(t) d t\right] \\
= & \|E[F]\|_{H}^{2}+E\left[\int_{a}^{b} \sum_{i, k=1}^{\infty} v_{i k}^{2}(t) d t\right] \\
= & \|E[F]\|_{H}^{2}+E\left[\int_{a}^{b}\|\varphi(t)\|_{\mathscr{L}_{2}(\mathbb{B}, H)}^{2} d t\right],
\end{aligned}
$$

where

$$
\varphi(t):=\sum_{i, \mathrm{k}=1}^{\infty} v_{i k}(t)\left(g_{i} \otimes e_{k}\right)
$$

By the polarization identity, we obtain the following assertion.

Corollary 2. Let $\xi(t)$ and $\eta(t)$ be $\mathscr{L}_{2}(\mathbb{H}, H)$-valued random processes, adapted to the filtration $\mathscr{B}_{t}^{a}, a \leq t \leq b$, such that

$$
\int_{a}^{b} E\left[\|\xi(t)\|_{\mathscr{L}_{2}(\mathbb{\boxplus}, H)}^{2}\right]<\infty, \quad \int_{a}^{b} E\left[\|\eta(t)\|_{\mathscr{L}_{2}(\mathbb{M}, H)}^{2}\right]<\infty .
$$

Then,

$$
\begin{gathered}
E\left[\left(\int_{a}^{b} \xi(t) d W(t), \int_{a}^{b} \eta(t) d W(t)\right)_{H}\right] \\
=\int_{a}^{b} E\left[(\xi(t), \eta(t))_{\mathscr{L}_{2}(\mathbb{E}, H)}\right] d t .
\end{gathered}
$$

\section{Multiple Itô Integrals with respect to the Cylindrical Wiener Process}

Let $\xi\left(t_{1}, t_{2}, \omega\right)$ be a function defined for $a \leq t_{1} \leq t_{2} \leq b$, $\omega \in \Omega$ taking values in the space $\mathscr{L}_{2}\left(\mathbb{H} ; \mathscr{L}_{2}(\mathbb{H} ; H)\right)$ and $\mathscr{B}\left(\mathbb{R}^{2}\right) \times \mathscr{F}$-measurable. If for any $t_{2} \in[a ; b]$ the random process $\xi\left(t, t_{2}\right), a \leq t \leq t_{2}$ is adapted to the filtration $\mathscr{B}_{t}^{a}, 0 \leq$ $t \leq t_{2}$ and the condition

$$
E\left[\int_{a}^{t_{2}}\left\|\xi\left(t, t_{2}\right)\right\|_{\mathscr{L}_{2}\left(\mathbb{H} ; \mathscr{L}_{2}(\mathbb{\boxplus} ; H)\right)}^{2} d t\right]<\infty
$$

holds true, then the Itô integral

$$
\int_{a}^{t_{2}} \xi\left(t_{1}, t_{2}\right) d W\left(t_{1}\right)
$$

is well defined and is an element of the space $L^{2}\left(\Omega ; \mathscr{L}_{2}(\mathbb{H} ; H)\right)$ for all $t_{2} \in[a ; b]$. As a function of $t_{2}$, it is an $\mathscr{L}_{2}(\mathbb{H} ; H)$-valued random process adapted to the filtration $\mathscr{B}_{t}^{a}$. We also have

$$
\begin{aligned}
& E\left[\left\|\int_{a}^{t_{2}} \xi\left(t_{1}, t_{2}\right) d W\left(t_{1}\right)\right\|_{\mathscr{L}_{2}(\mathbb{E} ; H)}^{2}\right]
\end{aligned}
$$

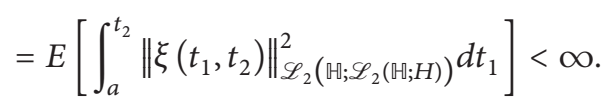

If moreover we have

$$
E\left[\int_{a}^{b} \int_{a}^{t_{2}}\left\|\xi\left(t_{1}, t_{2}\right)\right\|_{\mathscr{L}_{2}\left(\mathbb{M} ; \mathscr{L}_{2}(\mathbb{M} ; H)\right)}^{2} d t_{1} d t_{2}\right]<\infty,
$$

then the following iterated Itô integral is well defined:

$$
\int_{a}^{b} \int_{a}^{t_{2}} \xi\left(t_{1}, t_{2}\right) d W\left(t_{1}\right) d W\left(t_{2}\right)
$$


and it satisfies the equality

$$
\begin{gathered}
E\left[\left\|\int_{a}^{b} \int_{a}^{t_{2}} \xi\left(t_{1}, t_{2}\right) d W\left(t_{1}\right) d W\left(t_{2}\right)\right\|_{H}^{2}\right] \\
=E\left[\int_{a}^{b} \int_{a}^{t_{2}}\left\|\xi\left(t_{1}, t_{2}\right)\right\|_{\mathscr{L}_{2}(\mathbb{W} ; H)}^{2} d t_{1} d t_{2}\right] .
\end{gathered}
$$

Note that the operators $g_{i} \otimes e_{j} \otimes e_{k}$ defined by the equality

$$
\left(g_{i} \otimes e_{j} \otimes e_{k}\right) x:=\left(g_{i} \otimes e_{j}\right)\left(e_{k}, x\right)_{\mathbb{\square}}, \quad i, j, k \in \mathbb{N}
$$

form an orthonormal basis in the space $\mathscr{L}_{2}\left(\mathbb{H} ; \mathscr{L}_{2}(\mathbb{H} ; H)\right)$ and any operator $A \in \mathscr{L}_{2}\left(\mathbb{M} ; \mathscr{L}_{2}(\mathbb{H} ; H)\right)$ has the following decomposition:

$$
\begin{aligned}
& A=\sum_{i, j, k} a_{i j k}\left(g_{i} \otimes e_{j} \otimes e_{k}\right), \\
& \|A\|_{\mathscr{L}_{2}\left(\mathbb{\square} ; \mathscr{L}_{2}(\mathbb{\square} ; H)\right)}^{2}=\sum_{i, j, k} a_{i j k}^{2}<\infty .
\end{aligned}
$$

We can identify the decomposition (34) with the $H$ valued bilinear form on $\mathbb{H} \times \mathbb{H}$ defined by the equality $\left(g_{i} \otimes e_{j} \otimes e_{k}\right)(x, y):=g_{i}\left(e_{j}, x\right)_{\mathbb{U}}\left(e_{k}, y\right)_{\mathbb{\square}}$. Thus, the space $\mathscr{L}_{2}\left(\mathbb{H} ; \mathscr{L}_{2}(\mathbb{H} ; H)\right)$ can be identified with the Hilbert space $\mathscr{L}_{2}^{2}(\mathbb{M} \times \mathbb{M} ; H)$ of all continuous bilinear forms $A$ of the form (34) with the norm generated by the scalar product

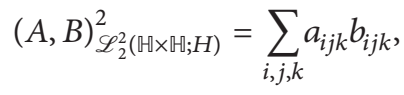

where $A=\sum_{i, j, k} a_{i j k}\left(g_{i} \otimes e_{j} \otimes e_{k}\right), B=\sum_{i, j, k} b_{i j k}\left(g_{i} \otimes e_{j} \otimes\right.$ $\left.e_{k}\right)$. So, the iterated integral (31) with respect to the cylindrical Wiener process $W(t)$ is well defined for any $\mathscr{L}_{2}^{2}(\mathbb{M} \times \mathbb{M} ; H)$ valued $\mathscr{B}\left(\mathbb{R}^{2}\right) \times \mathscr{F}$-measurable function $\xi\left(t_{1}, t_{2}, \omega\right)$, defined on $S_{2}^{a, b} \times \Omega$, where

$$
S_{2}^{a, b}=\left\{\left(t_{1}, t_{2}\right) \mid a \leq t_{1} \leq t_{2} \leq b\right\},
$$

if it satisfies the condition (30).

One can easily extend the above definition to the case of arbitrary $n>2$, defining the $n$ times iterated integral

$$
\begin{aligned}
& J_{n}(\xi) \\
& \quad=\int_{a}^{b} \int_{a}^{t_{2}} \ldots \int_{a}^{t_{n-1}} \xi\left(t_{1}, t_{2}, \ldots, t_{n}, \omega\right) d W\left(t_{1}\right), \ldots, d W\left(t_{n}\right)
\end{aligned}
$$

inductively for any function $\xi: S_{n} \times \Omega \rightarrow \mathscr{L}_{2}^{n}\left(\mathbb{H}^{\times n} ; H\right)$, where

$$
S_{n}^{a, b}=\left\{\left(t_{1}, \ldots, t_{n}\right) \mid a \leq t_{1} \leq, \ldots, t_{n} \leq b\right\},
$$

$\mathscr{L}_{2}^{n}\left(\mathbb{M}^{\times n} ; H\right)$ is the space of all continuous $H$-valued $n$-linear forms on $\mathbb{W} \times \cdots \times \mathbb{W}$ having form

$$
A=\sum_{i, k_{1}, \ldots, k_{n}} a_{i, k_{1}, \ldots, k_{n}}\left(g_{i} \otimes e_{k_{1}} \otimes, \ldots, \otimes e_{k_{n}}\right)
$$

such that

$$
\|A\|_{\mathscr{L}_{2}^{n}\left(\mathbb{\mathbb { H } ^ { \times n }} ; H\right)}^{2}=\sum_{i, k_{1}, \ldots, k_{n}} a_{i, k_{1}, \ldots, k_{n}}^{2}<\infty,
$$

if it satisfies the following conditions:

(n-i) $\xi$ is $\mathscr{B}\left(\mathbb{R}^{n}\right) \times \mathscr{F}$-measurable;

(n-ii) $\xi\left(t_{1}, t_{2}, \ldots, t_{n}\right)$ is $\mathscr{B}_{t_{1}}^{a}$-measurable for any $\left(t_{1}, t_{2}, \ldots, t_{n}\right) \in S_{n}^{a, b}$ (n-iii) $\begin{aligned} E\left[\int_{a}^{b} \int_{a}^{t_{2}} \cdots \int_{a}^{t_{n-1}}\left\|\xi\left(t_{1}, \ldots, t_{n}\right)\right\|_{L_{2}^{n}\left(H^{\times n} ; H\right)}^{2} d t_{1}, \ldots, d t_{n}\right]< \\ \infty\end{aligned}$

For the defined iterated integral, we have

$$
\begin{aligned}
& E\left[\left\|J_{n}(\xi)\right\|_{H}^{2}\right] \\
& =\int_{a}^{b} \int_{a}^{t_{2}} \ldots \int_{a}^{t_{n-1}} E\left[\left\|\xi\left(t_{1}, \ldots, t_{n}\right)\right\|_{\mathscr{L}_{2}^{n}\left(\mathbb{Q}^{\times n} ; H\right)}^{2}\right] d t_{1}, \ldots, d t_{n} .
\end{aligned}
$$

We state without proof the next two lemmas which are straightforward generalizations of the corresponding properties of the $\mathbb{R}$-valued iterated Itô integrals.

Lemma 3. Let $\xi: S_{n_{1}}^{a, b} \times \Omega \rightarrow \mathscr{L}_{2}^{n_{1}}\left(\mathbb{W}^{\times n_{1}} ; H\right)$ and $\eta: S_{n_{2}}^{a, b} \times$ $\Omega \rightarrow \mathscr{L}_{2}^{n_{2}}\left(\mathbb{H}^{\times n_{2}} ; H\right)$ satisfy the conditions (n-i), (n-ii) and (niii) with $n=n_{1}$ and $n=n_{2}$ correspondingly. Then,

$$
E\left[\left(J_{n_{1}}(\xi), J_{n_{2}}(\eta)\right)_{H}\right]= \begin{cases}0, & n_{1} \neq n_{2}, \\ (\xi, \eta)_{L^{2}\left(S_{n}^{a, b} ; \mathscr{L}_{2}^{n}(\mathbb{M} \times n ; H)\right)} & \\ n_{1}=n_{2}=n . & \end{cases}
$$

Lemma 4. Let $\xi: S_{n}^{a, b} \times \Omega \rightarrow \mathscr{L}_{2}^{n}\left(\mathbb{M}^{\times n} ; H\right)$ satisfy the conditions (n-i), (n-ii) and (n-iii). Then,

$$
\begin{aligned}
& E\left[J_{n}(\xi) \mid \mathscr{B}_{t}^{a, b}\right] \\
& =\int_{a}^{t} \int_{a}^{t_{2}} \ldots \int_{a}^{t_{n-1}} \xi\left(t_{1}, t_{2}, \ldots, t_{n}, \omega\right) d W\left(t_{1}\right), \ldots, d W\left(t_{n}\right) .
\end{aligned}
$$

Denote by $\widehat{L}^{2}\left([a ; b]^{n} ; \mathscr{L}_{2}^{n}\left(\mathbb{M}^{\times n} ; H\right)\right)$ the space of all symmetric functions $f \in:[a ; b]^{n} \rightarrow \mathscr{L}_{2}^{n}\left(\mathbb{H}^{\times n} ; H\right)$ satisfying the condition

$$
\begin{aligned}
& \|f\|_{L^{2}\left([a ; b]^{n} ; \mathscr{L}_{2}^{n}\left(\mathbb{M}^{\times n} ; H\right)\right)}^{2} \\
& =\int \underset{[a ; b]^{n}}{\ldots} \int\left\|f_{n}\left(t_{1}, \ldots, t_{n}\right)\right\|_{\mathscr{L}_{2}^{n}\left(\mathbb{\boxplus}^{\times n} ; H\right)}^{2} d t_{1}, \ldots, d t_{n}<\infty .
\end{aligned}
$$

Definition 5. For any $f \in \widehat{L}^{2}\left([a ; b]^{n} ; \mathscr{L}_{2}^{n}\left(\mathbb{H}^{\times n} ; H\right)\right)$, define the multiple $n$-fold Itô integral by the equality

$$
I_{n}(f):=n ! J_{n}(f),
$$

if the right-hand side iterated integral exists. 


\section{The Decomposition Theorem}

To establish the main result, we need a few lemmas.

Lemma 6. The set of random variables

$$
\begin{aligned}
& \left\{\varphi\left(\beta_{j_{1}}\left(t_{i_{1}}\right), \ldots, \beta_{j_{n}}\left(t_{i_{n}}\right)\right) \mid j_{k} \in \mathbb{N}, t_{k} \in[a ; b],\right. \\
& \left.\varphi \in C_{0}^{\infty}\left(\mathbb{R}^{n}\right), n \in \mathbb{N}\right\}
\end{aligned}
$$

is dense in $L^{2}\left(\mathscr{B}_{b}^{a}, P\right)$.

Proof. Let $\left\{t_{i}\right\}_{i=1}^{\infty}$ be a dense subset in $[a ; b]$. Let $\left\{\zeta_{k}\right\}_{k=1}^{\infty}$ be a fixed ordering of the countable set of random variables $\left\{\beta_{j}\left(t_{i}\right)\right\}_{j, i=1}^{\infty}$. Denote by $\mathscr{H}_{p}$ be the $\sigma$-algebra generated by $\left\{\zeta_{k}\right\}_{k=1}^{p}$. We have $\mathscr{H}_{p} \subset \mathscr{H}_{p+1}$ for all $p \in \mathbb{N}$ and $\mathscr{B}_{b}^{a}$ is the smallest $\sigma$-algebra containing all $\mathscr{H}_{p}$.

For any $g \in L^{2}\left(\mathscr{B}_{b}^{a}, P\right)$, we have

$$
g=E\left(g \mid \mathscr{B}_{b}^{a}\right)=\lim _{p \rightarrow \infty} E\left(g \mid \mathscr{H}_{p}\right),
$$

where the limit is pointwise a.e. with respect to $P$ and in $L^{2}\left(\mathscr{B}_{b}^{a}, P\right)$. By the Doob-Dynkin lemma for any $p \in \mathbb{N}$, there exists a Borel function $g_{p}: \mathbb{R}^{p} \rightarrow \mathbb{R}$ such that

$$
E\left[g \mid \mathscr{H}_{p}\right]=g_{p}\left(\zeta_{1}, \ldots, \zeta_{p}\right) .
$$

Let $P_{\zeta_{1}, \ldots, \zeta_{p}}$ be the probability measure on $\mathbb{R}^{p}$ generated by $\zeta_{1}, \ldots, \zeta_{p}$. Since $g_{p}$ can be approximated in $L^{2}\left(\mathbb{R}^{p}, P_{\zeta_{1}, \ldots, \zeta_{p}}\right)$ by functions $\varphi \in C_{0}^{\infty}\left(\mathbb{R}^{p}\right)$, the assertion follows.

Consider the following exponential functionals of Brownian motions $\beta_{j}$ :

$$
\begin{array}{r}
\mathscr{E}_{\theta}^{j, a, b}:=\exp \left[\int_{a}^{b} \theta(t) d \beta_{j}(t)-\frac{|\theta|_{0}^{2}}{2}\right], \\
j \in \mathbb{N}, \theta \in L^{2}[a ; b] .
\end{array}
$$

Lemma 7. The linear span of the set

$$
\left\{\prod_{k=1}^{p} \mathscr{E}_{\theta_{k}}^{j_{k}, a, b} \mid j_{k} \in \mathbb{N}, \theta_{k} \in L^{2}[a ; b], k=1, \ldots, p, p \in \mathbb{N}\right\}
$$

is dense in $L^{2}\left(\mathscr{B}_{b}^{a}, P\right)$.

Proof. Let $g \in L^{2}\left(\mathscr{B}_{b}^{a}, P\right)$ be orthogonal to all functions of the family (50). Take $\theta_{k}(t)=\lambda_{k} 1_{\left[a ; t_{k}\right]}$, where $\left\{t_{k}\right\}_{k=1}^{p} \subset[a ; b]$, $\bar{\lambda}=\left(\lambda_{1}, \ldots, \lambda_{p}\right) \in \mathbb{R}^{p}$. Then, we have

$$
\begin{aligned}
& \prod_{k=1}^{p} \mathscr{E}_{\theta_{k}, a, b}^{j_{k}} \\
& \quad=\prod_{k=1}^{p} \exp \left[\lambda_{k} \int_{a}^{t_{k}} d \beta_{j_{k}}(t)-\frac{t_{k}-a}{2}\right] \\
& \quad=\exp \left[-\frac{1}{2} \sum_{k=1}^{p}\left(t_{k}-a\right)\right] \cdot \exp \left[\sum_{k=1}^{p} \lambda_{k} \beta_{j_{k}}\left(t_{k}\right)\right] .
\end{aligned}
$$

It follows that

$$
G(\bar{\lambda}):=\int_{\Omega} \exp \left[\sum_{k=1}^{p} \lambda_{k} \beta_{j_{k}}\left(t_{k}\right)\right] g(\omega) d P(\omega)=0
$$

for all $p \in \mathbb{N}, \bar{\lambda} \in \mathbb{R}^{p}, j_{1}, \ldots, j_{p} \in \mathbb{N}, t_{1}, \ldots, t_{p} \in[a ; b]$.

Fix $p, j_{1}, \ldots, j_{p}, t_{1}, \ldots, t_{p}$. The corresponding function $G(\bar{\lambda})$ is real analytic. Consider its analytic extension onto $\mathbb{C}^{p}$. It is also equal to zero. Taking $\bar{\lambda}=\left(i y_{1}, \ldots, i y_{p}\right)$, we obtain

$$
\int_{\Omega} \exp \left[i \sum_{k=1}^{p} y_{k} \beta_{j_{k}}\left(t_{k}\right)\right] g(\omega) d P(\omega)=0
$$

For any $\varphi \in C_{0}^{\infty}\left(\mathbb{R}^{p}\right)$ denoting by $\widehat{\varphi}$ its Fourier transform, we obtain

$$
\begin{aligned}
& \int_{\Omega} \varphi\left(\beta_{j_{1}}\left(t_{1}\right), \ldots, \beta_{j_{p}}\left(t_{p}\right)\right) g(\omega) d P(\omega) \\
& =\int_{\Omega} \frac{1}{(2 \pi)^{p / 2}} \\
& \quad \times \int_{\mathbb{R}^{p}} \widehat{\varphi}(\bar{y}) \exp \left[i \sum_{k=1}^{p} y_{k} \beta_{j_{k}}\left(t_{k}\right)\right] d \bar{y} g(\omega) d P(\omega) \\
& =\frac{1}{(2 \pi)^{p / 2}} \int_{\mathbb{R}^{p}} \hat{\varphi}(\bar{y}) \\
& \quad \times \int_{\Omega} \exp \left[i \sum_{k=1}^{p} y_{k} \beta_{j_{k}}\left(t_{k}\right)\right] g(\omega) d P(\omega) d \bar{y}=0 .
\end{aligned}
$$

Thus, $g$ is orthogonal to a dense subset in $L^{2}\left(\mathscr{B}_{b}^{a}, P\right)$.

Lemma 8. For any $\alpha_{1}, \ldots, \alpha_{n} \in \mathbb{N} \cup\{0\}, \theta_{1}, \ldots, \theta_{n} \in \mathcal{S}$, the product

$$
\prod_{j=1}^{n} h_{\alpha_{j}}\left(\frac{\int_{a}^{b} \theta_{j}(t) d \beta_{j}(t)}{\left|\theta_{j} \cdot 1_{[a ; b]}\right|_{0}}\right)
$$

is a linear combination of the iterated Itô integrals of the form

$$
\int_{a}^{b} \int_{a}^{t_{p}} \ldots \int_{a}^{t_{2}} \theta_{j_{1}}\left(t_{1}\right), \ldots, \theta_{j_{p}}\left(t_{p}\right) d \beta_{j_{1}}\left(t_{1}\right), \ldots, d \beta_{j_{p}}\left(t_{p}\right),
$$

where $p=\alpha_{1}+\cdots+\alpha_{n}$ and $j_{1}, \ldots, j_{p} \in\{1, \ldots, n\}$. 
Proof. We use induction with respect to $p$. The assertion is evident for $p=1$. Suppose it is true for some $p \in \mathbb{N}$. Let $\alpha_{1}+\cdots+\alpha_{n}=p+1$. We introduce the following notation:

$$
\begin{aligned}
X_{j}(t) & :=\frac{\left|\theta_{j} \cdot 1_{[a ; t]}\right|_{0}^{\alpha_{j}}}{\alpha_{j} !} h_{\alpha_{j}}\left(\frac{\int_{a}^{t} \theta_{j}(\tau) d \beta_{j}(\tau)}{\left|\theta_{j} \cdot 1_{[a ; t]}\right|_{0}}\right) \\
& =\int_{a}^{t} \int_{a}^{t_{\alpha_{j}}} \ldots \\
& \quad \int_{a}^{t_{2}} \theta_{j}\left(t_{1}\right), \ldots, \theta_{j}\left(t_{\alpha_{j}}\right) d \beta_{j}\left(t_{1}\right), \ldots, d \beta_{j}\left(t_{\alpha_{j}}\right) \\
= & \int_{a}^{t} Y_{j}\left(t_{\alpha_{j}}, \omega\right) d \beta_{j}\left(t_{\alpha_{j}}\right), \quad j=1, \ldots, n .
\end{aligned}
$$

This means that $X_{j}$ satisfy the following stochastic differential equations:

$$
d X_{j}(t)=Y_{j}(t, \omega) d \beta_{j}\left(t_{\alpha_{j}}\right), \quad j=1, \ldots, n,
$$

and we have

$$
Y_{j}(t)=\frac{\left|\theta_{j} \cdot 1_{[a ; t]}\right|_{0}^{\alpha_{j}-1}}{\left(\alpha_{j}-1\right) !} h_{\alpha_{j}-1}\left(\frac{\int_{a}^{t} \theta_{j}(\tau) d \beta_{j}(\tau)}{\left|\theta_{j} \cdot 1_{[a ; t]}\right|_{0}}\right) .
$$

By the Itô formula, we obtain

$$
\begin{aligned}
d\left(\prod_{j=1}^{n} X_{j}(t)\right)= & \sum_{j=1}^{n}\left[\prod_{i \neq j} X_{i}(t)\right] d X_{j}(t) \\
& +\sum_{i \neq j} d X_{i}(t) \cdot d X_{j}(t) .
\end{aligned}
$$

Since $d X_{i}(t) \cdot d X_{j}(t)=Y_{i}(t, \omega) Y_{j}(t, \omega) d \beta_{i}(t) d \beta_{j}(t)=0$, we come to the equality

$$
\begin{aligned}
& \prod_{j=1}^{n} X_{j}(b) \\
& =\sum_{j=1}^{n} \int_{a}^{b}\left[\prod_{i \neq j} X_{i}(t)\right] Y_{j}(t, \omega) d \beta_{j}(t) \\
& =\sum_{j=1}^{n} \int_{a}^{b}\left[\prod_{i \neq j} \frac{\left|\theta_{j} \cdot 1_{[a ; t]}\right|_{0}^{\alpha_{j}}}{\alpha_{j}} h_{\alpha_{j}}\left(\int_{a}^{t} \theta_{j}(\tau) d \beta_{j}(\tau)\right)\right. \\
& \left.\quad \frac{\left|\theta_{j} \cdot 1_{[a ; t]}\right|_{0}^{\alpha_{j}-1}}{\left(\alpha_{j}-1\right) !} h_{\alpha_{j}-1}\left(\frac{\int_{a}^{t} \theta_{j}(\tau) d \beta_{j}(\tau)}{\left|\theta_{j} \cdot 1_{[a ; t]}\right|_{0}}\right)\right] \\
& \times \theta_{j}(t) d \beta_{j}(t) .
\end{aligned}
$$

Since $\sum_{i \neq j} \alpha_{i}+\alpha_{j}-1=p$, we can apply the assumption to the products in the brackets, thus completing the proof.
Theorem 9. Any $\mathscr{B}_{b}^{a}$-measurable random variable $F \in$ $L^{2}(\Omega ; H)$ has the unique decomposition

$$
F=\sum_{n=0}^{\infty} I_{n}\left(f_{n}\right)
$$

where $f_{n} \in \widehat{L}^{2}\left([a ; b]^{n} ; \mathscr{L}_{2}^{n}\left(\mathbb{\boxplus}^{\times n} ; H\right)\right), n \in \mathbb{N}, I_{0}\left(f_{0}\right):=f_{0} \in H$. It holds that

$$
\|F\|_{L^{2}(\Omega ; H)}^{2}=\sum_{n=0}^{\infty} n !\left\|f_{n}\right\|_{L^{2}\left([a ; b]^{n} ; \mathscr{L}_{2}^{n}\left(\mathbb{\boxplus}^{\times n} ; H\right)\right)}^{2},
$$

where $\left\|f_{0}\right\|_{L^{2}\left([a ; b]^{0} ; \mathscr{L}_{2}^{0}\left(\mathbb{H}^{\times 0} ; H\right)\right)}^{2}:=\left\|f_{0}\right\|_{H}^{2}$.

Proof. By Theorem 1, we have

$$
F=E[F]+\int_{a}^{b} \varphi_{1}\left(t_{1}, \omega\right) d W\left(t_{1}\right),
$$

where $\varphi_{1}(t)$ is a $\mathscr{L}_{2}^{1}(\mathbb{1} ; H)$-valued random variable, $\mathscr{B}_{t}^{a}$ measurable for any $t$. For its norm, we have

$$
\|F\|_{L^{2}(\Omega ; H)}^{2}=\|E[F]\|_{H}^{2}+E\left[\int_{a}^{b}\left\|\varphi_{1}(t)\right\|_{\mathscr{L}_{2}(\mathrm{H} ; H)}^{2} d t_{1}\right] .
$$

Let $\psi_{0}:=E[F]$. For any $t_{1} \in[a, b]$ by Theorem 1 , taking $t_{1}$ for $b$ and $\mathscr{L}_{2}(\mathbb{I} ; H)$ for $H$, we obtain the following representation:

$$
\varphi_{1}\left(t_{1}\right)=E\left[\varphi_{1}\left(t_{1}\right)\right]+\int_{a}^{t_{1}} \varphi_{2}\left(t_{1}, t_{2}, \omega\right) d W\left(t_{2}\right),
$$

where $\varphi_{2}: S_{2}^{a, b} \times \Omega \rightarrow \mathscr{L}_{2}\left(\mathbb{\square} ; \mathscr{L}_{2}(\mathbb{M} ; H)\right) \cong \mathscr{L}_{2}^{2}\left(\mathbb{\boxplus}^{\times 2} ; H\right)$, with the following equality for the norm:

$$
\begin{aligned}
\left\|\varphi_{1}\left(t_{1}\right)\right\|_{\mathscr{L}_{2}\left(\Omega ; \mathscr{L}_{2}(\mathbb{H} ; H)\right)}^{2}= & \left\|E\left[\varphi_{1}\left(t_{1}\right)\right]\right\|_{\mathscr{L}_{2}(\mathbb{H} ; H)}^{2} \\
& +E\left[\int_{a}^{t_{1}}\left\|\varphi_{2}\left(t_{1}, t_{2}\right)\right\|_{\mathscr{L}_{2}^{2}\left(\mathbb{H}^{\times 2} ; H\right)}^{2} d t_{2}\right] .
\end{aligned}
$$

Set $\psi_{1}\left(t_{1}\right):=E\left[\varphi_{1}\left(t_{1}\right)\right]$. Substituting the representation (66) into (64), we come to the equality

$$
\begin{aligned}
F= & J_{0}\left(\psi_{0}\right)+\int_{a}^{b} \psi_{1}\left(t_{1}\right) d W\left(t_{1}\right) \\
& +\int_{a}^{b} \int_{a}^{t_{1}} \varphi_{2}\left(t_{1}, t_{2}, \omega\right) d W\left(t_{2}\right) d W\left(t_{1}\right) .
\end{aligned}
$$

From (65) and (67), it follows that

$$
\begin{aligned}
& \|F\|_{L^{2}(\Omega ; H)}^{2}=\left\|\psi_{0}\right\|_{H}^{2}+\int_{a}^{b}\left\|\psi_{1}\left(t_{1}\right)\right\|_{\mathscr{L}_{2}^{1}(\boxplus ; H)}^{2} d t_{1} \\
& +E\left[\int_{a}^{b} \int_{a}^{t_{1}}\left\|\varphi_{2}\left(t_{1}, t_{2}\right)\right\|_{\mathscr{L}_{2}^{2}\left(\uplus^{\times 2} ; H\right)}^{2} d t_{2} d t_{1}\right] \\
& =\left\|\psi_{0}\right\|_{H}^{2}+\int_{a}^{b}\left\|\psi_{1}\left(t_{1}\right)\right\|_{\mathscr{L}_{2}^{1}(\uplus ; H)}^{2} d t_{1} \\
& +\int_{a}^{b} \int_{a}^{t_{1}} E\left[\left\|\varphi_{2}\left(t_{1}, t_{2}\right)\right\|_{\mathscr{L}_{2}^{2}\left(\mathbb{H}^{\times 2} ; H\right)}^{2}\right] d t_{2} d t_{1} .
\end{aligned}
$$


Applying further in similar manner Theorem 1 and setting

$$
\begin{array}{r}
\psi_{n}\left(t_{1}, \ldots, t_{n}\right):=E\left[\varphi_{n}\left(t_{1}, \ldots, t_{n}\right)\right] \in \mathscr{L}_{2}^{n}\left(\mathbb{M}^{\times n} ; \mathrm{H}\right), \\
\left(t_{1}, \ldots, t_{n}\right) \in S_{n}^{a, b}
\end{array}
$$

at the $n$th step of this process, we obtain

$$
F=J_{0}\left(\psi_{0}\right)+J_{1}\left(\psi_{1}\right)+\cdots+J_{n}\left(\psi_{n}\right)+J_{n+1}\left(\varphi_{n+1}\right)
$$

with

$$
\begin{aligned}
& \|F\|_{L^{2}(\Omega ; H)}^{2} \\
& =\left\|\psi_{0}\right\|_{H}^{2}+\int_{a}^{b}\left\|\psi_{1}\left(t_{1}\right)\right\|_{\mathscr{L}_{2}^{1}(\mathbb{M} ; H)}^{2} d t_{1} \\
& +\int_{a}^{b} \int_{a}^{t_{1}}\left\|\psi_{2}\left(t_{1}, t_{2}\right)\right\|_{\mathscr{L}_{2}^{2}\left(\mathbb{\boxplus}^{\times 2} ; H\right)}^{2} d t_{2} d t_{1} \\
& +\cdots+\int_{a}^{b} \int_{a}^{t_{1}} \cdots \\
& \int_{a}^{t_{n-1}}\left\|\psi_{n}\left(t_{1}, \ldots, t_{n}\right)\right\|_{\mathscr{L}_{2}^{n}\left(\mathbb{\mathbb { M } ^ { \times n }} ; H\right)}^{2} d t_{n}, \ldots, d t_{1} \\
& +\int_{a}^{b} \int_{a}^{t_{1}} \cdots \\
& \left.\int_{a}^{t_{n}} E\left[\left\|\varphi_{n+1}\left(t_{1}, \ldots, t_{n+1}\right)\right\|_{\mathscr{L}_{2}^{n+1}(\mathbb{M} \times(n+1)}^{2} ; H\right)\right] d t_{n+1}, \ldots, d t_{1} .
\end{aligned}
$$

It follows from here that

$\left\|\psi_{0}\right\|_{H}^{2}$

$$
\begin{aligned}
+\sum_{k=1}^{n} \int_{a}^{b} \int_{a}^{t_{1}} & \ldots \\
& \quad \int_{a}^{t_{k-1}}\left\|\psi_{k}\left(t_{1}, \ldots, t_{k}\right)\right\|_{\mathscr{L}_{2}^{k}(\mathbb{\square} \times k ; H)}^{2} d t_{k}, \ldots, d t_{1}
\end{aligned}
$$$$
\leq\|F\|_{L^{2}(\Omega ; H)}^{2}
$$

and consequently

$$
\begin{aligned}
& \left\|\psi_{0}\right\|_{H}^{2} \\
& +\sum_{k=1}^{\infty} \int_{a}^{b} \int_{a}^{t_{1}} \ldots \\
& \quad \int_{a}^{t_{k-1}}\left\|\psi_{k}\left(t_{1}, \ldots, t_{k}\right)\right\|_{\mathscr{L}_{2}^{k}(\mathbb{\square} \times k ; H)}^{2} d t_{k}, \ldots, d t_{1}
\end{aligned}
$$

$<\infty$.

This means that the series

$$
\sum_{n=0}^{\infty} J_{n}\left(\psi_{n}\right)
$$

is convergent in the space $L^{2}(\Omega ; H)$ and

$$
\Phi:=\lim _{n \rightarrow \infty} J_{n+1}\left(\varphi_{n+1}\right)
$$

is an element of this space. By Lemma 3, the integral $J_{n+1}\left(\varphi_{n+1}\right)$ is orthogonal in $L^{2}(\Omega ; H)$ to the integrals $J_{0}\left(\psi_{0}\right), \ldots, J_{n}\left(\psi_{n}\right)$ for all $\psi_{0} \in H, \psi_{1} \in L^{2}\left(S_{1}^{a, b} ; \mathscr{L}_{2}^{1}(\mathbb{H} ; H)\right)$, $\ldots, \psi_{n} \in L^{2}\left(S_{n}^{a, b} ; \mathscr{L}_{2}^{n}\left(\mathbb{M}^{\times n} ; H\right)\right)$. It follows that $\Phi$ is orthogonal to $J_{n}\left(\psi_{n}\right)$ for all $n \in \mathbb{N} \cup\{0\}, \psi_{n} \in L^{2}\left(S_{n}^{a, b} ; \mathscr{L}_{2}^{n}\left(\mathbb{M}^{\times n} ; H\right)\right)$.

Note that by the well-known connection between iterated Itô integrals with respect to a Brownian motion and the Hermite polynomials, we have

$$
\begin{aligned}
J_{n} & \left(g_{i} \otimes e_{j}^{\otimes n} \theta^{\otimes n}\right) \\
& =g_{i} \int_{a}^{b} \int_{a}^{t_{1}} \ldots \int_{a}^{t_{n-1}} \theta^{\otimes n}\left(t_{1}, \ldots, t_{n}\right) d \beta_{j}\left(t_{n}\right) \ldots d \beta_{j}\left(t_{1}\right) \\
& =g_{i}\left|\theta \cdot 1_{[a ; b]}\right|_{0}^{n} h_{n}\left(\frac{\int_{a}^{b} \theta(t) d \beta_{j}(t)}{\left|\theta \cdot 1_{[a ; b]}\right|_{0}}\right)
\end{aligned}
$$

for any $n, i, j \in \mathbb{N}, \theta \in \mathcal{S}$.

Let $\Phi=\sum_{i=1}^{\infty} \Phi_{i} g_{i}$, where $\Phi_{i} \in L^{2}(\Omega ; \mathbb{R})$. For any $n, i, j_{1}, \ldots, j_{n} \in \mathbb{N}$ and any $\theta_{j_{1}}, \ldots, \theta_{j_{n}} \in \mathcal{S}$, we have

$$
\begin{aligned}
& E\left[\Phi, \cdot \int_{a}^{b} \int_{a}^{t_{n}} \ldots\right. \\
& \left.\quad \int_{a}^{t_{2}} \theta_{j_{1}}\left(t_{1}\right), \ldots, \theta_{j_{n}}\left(t_{n}\right) d \beta_{j_{1}}\left(t_{1}\right), \ldots, d \beta_{j_{n}}\left(t_{n}\right)\right] \\
& =E\left[\left(\Phi, g_{i} \int_{a}^{b} \int_{a}^{t_{n}} \ldots\right.\right. \\
& \left.\left.\quad \int_{a}^{t_{2}} \theta_{j_{1}}\left(t_{1}\right), \ldots, \theta_{j_{n}}\left(t_{n}\right) d \beta_{j_{1}}\left(t_{1}\right), \ldots, d \beta_{j_{n}}\left(t_{n}\right)\right)_{H}\right] \\
& =\left(\Phi, J_{n}\left(g_{i} \otimes e_{j_{1}} \otimes \cdots \otimes e_{j_{n}} \otimes \theta_{j_{1}} \otimes \cdots \otimes \theta_{j_{n}}\right)\right)=0,
\end{aligned}
$$

from where, by Lemma 8 and the equality (77), it follows that, for any $i \in \mathbb{N}, \Phi_{i}$ is orthogonal in $L^{2}(\Omega ; \mathbb{R})$ to the products of the form

$$
\prod_{j=1}^{n} h_{\alpha_{j}}\left(\frac{\int_{a}^{b} \theta_{j}(t) d \beta_{j}(t)}{\left|\theta_{j} \cdot 1_{[a ; b]}\right|_{0}}\right) .
$$

It follows from here and the connection between the Hermite polynomials and general powers that

$$
E\left[\Phi_{i} \prod_{j=1}^{n}\left(\int_{a}^{b} \theta_{j}(t) d \beta_{j}(t)\right)^{\alpha_{j}}\right]=0 .
$$


It follows from the expansion

$$
\begin{aligned}
\mathscr{E}_{\theta}^{j, a, b} & =\exp \left[\int_{a}^{b} \theta(t) d \beta_{j}(t)-\frac{|\theta|_{0}^{2}}{2}\right] \\
& =e^{-\left(|\theta|_{0}^{2} / 2\right)} \sum_{k=0}^{\infty} \frac{\left(\int_{a}^{b} \theta(t) d \beta_{j}(t)\right)^{k}}{k !}
\end{aligned}
$$

that a product of the form

$$
\prod_{k=1}^{n} \mathscr{E}_{\theta_{k}}^{j_{k}, a, b}
$$

has a decomposition into the series of the products of the form

$$
\prod_{j=1}^{n}\left(\int_{a}^{b} \theta_{j}(t) d \beta_{j}(t)\right)^{\alpha_{j}}, \quad \alpha_{j} \in \mathbb{N} \cup\{0\}, \quad j=1, \ldots, n,
$$

convergent in the space $L^{2}(\Omega ; \mathbb{R})$. Consequently, $\Phi_{i}$ is orthogonal to any product of the form (82). By Lemma 7, this means that $\Phi_{i}=0$ for any $i \in \mathbb{N}$; that is, $\Phi=0$. Passing to the limit in the equality (71), we obtain

$$
F=\sum_{n=0}^{\infty} J_{n}\left(\psi_{n}\right)
$$

Setting the functions $\psi_{n}$ to be equal to zero on $[a ; b]^{n} \backslash S_{n}^{a, b}$ and defining $f_{n}:=\widehat{\psi}_{n}$, where $\widehat{\psi}_{n}$ is the symmetrization of $\psi_{n}$, we obtain (62). By the equality

$$
\begin{aligned}
& \left\|f_{n}\right\|_{L^{2}\left([a ; b]^{n} ; \mathscr{L}_{2}^{n}\left(\mathbb{\boxplus}^{\times n} ; H\right)\right)}^{2} \\
& =\int \underset{[a ; b]^{n}}{\ldots} \int\left\|f_{n}\left(t_{1}, \ldots, t_{n}\right)\right\|_{\mathscr{L}_{2}^{n}\left(\mathbb{\boxplus}^{\times n} ; H\right)}^{2} d t_{1}, \ldots, d t_{n} \\
& =n ! \int_{S_{n}^{a, b}}^{\ldots} \int\left\|f_{n}\left(t_{1}, \ldots, t_{n}\right)\right\|_{\mathscr{L}_{2}^{n}\left(\mathbb{Q}^{\times n} ; H\right)}^{2} d t_{1}, \ldots, d t_{n} \\
& =n ! \int_{S_{n}^{a, b}}^{\ldots} \int\left\|\frac{\psi_{n}\left(t_{1}, \ldots, t_{n}\right)}{n !}\right\|_{\mathscr{L}_{2}^{n}\left(\mathbb{\mathbb { A } ^ { \times n }} ; H\right)}^{2} d t_{1}, \ldots, d t_{n} \\
& =\frac{1}{n !} \int_{S_{n}^{a, b}} \ldots \int\left\|\psi_{n}\left(t_{1}, \ldots, t_{n}\right)\right\|_{\mathscr{L}_{2}^{n}\left(\mathbb{\mathbb { Q }} \times \mathbb{\boxplus}^{\times n} ; H\right)}^{2} d t_{1}, \ldots, d t_{n},
\end{aligned}
$$

passing to the limit in (72), we obtain (63).

Definition 10. For any $f \in \widehat{L}^{2}\left(\mathbb{R}^{n} ; \mathscr{L}_{2}^{n}\left(\mathbb{M}^{\times n} ; H\right)\right)$, define the $n$ fold Itô integral $I_{n}(f)$ by the equality (45), where the iterated integral

$$
\begin{aligned}
J_{n}(f)=\int_{-\infty}^{+\infty} \int_{-\infty}^{t_{2}} \ldots \\
\\
\quad \int_{-\infty}^{t_{n-1}} \xi\left(t_{1}, t_{2}, \ldots, t_{n}, \omega\right) d W\left(t_{1}\right), \ldots, d W\left(t_{n}\right)
\end{aligned}
$$

is understood as the limit of the integrals $J_{n}\left(f \cdot 1_{[-A ; A]^{n}}\right)$ at $A \rightarrow+\infty$ in the space $L^{2}(\Omega ; H)$ if the limit exists.
Corollary 11. Any $F \in L^{2}(\Omega ; H)$ can be uniquely decomposed into the series

$$
F=\sum_{n=0}^{\infty} I_{n}\left(f_{n}\right)
$$

where $f_{n} \in \widehat{L}^{2}\left(\mathbb{R}^{n} ; \mathscr{L}_{2}^{n}\left(\mathbb{M}^{\times n} ; H\right)\right), n \in \mathbb{N}, I_{0}\left(f_{0}\right):=f_{0} \in H$. The equality

$$
\|F\|_{L^{2}(\Omega ; H)}^{2}=\sum_{n=0}^{\infty} n !\left\|f_{n}\right\|_{L^{2}\left(\mathbb{R}^{n} ; \mathscr{L}_{2}^{n}\left(\mathbb{\boxplus}^{\times n} ; H\right)\right)}^{2}
$$

holds true, where $\left\|f_{0}\right\|_{L^{2}\left(\mathbb{R}^{0} ; \mathscr{L}_{2}^{0}(\mathbb{\cap} \times 0 ; H)\right)}^{2}:=\left\|f_{0}\right\|_{H}^{2}$.

Proof. Let $F_{N}=E\left[F \mid \mathscr{B}_{N}^{-N}\right], N \in \mathbb{N}$. For any $N \in \mathbb{N}$, it can be uniquely represented in the form

$$
F_{N}=\sum_{n=0}^{\infty} I_{n}\left(f_{N, n}\right)=\sum_{n=0}^{\infty} J_{n}\left(\psi_{N, n}\right)
$$

where $\psi_{N, n} \in L^{2}\left(S_{n}^{-N, N} ; \mathscr{L}_{2}^{n}\left(\mathbb{M}^{\times n} ; H\right)\right)$ and $f_{N, n}$ is the symmetrization of the function $\psi_{N, n}$ set to be equal to zero at the set $[-N ; N]^{n} \backslash S_{n}^{-N, N}$. For its norm, we have

$$
\begin{aligned}
\left\|F_{N}\right\|_{L^{2}(\Omega ; H)}^{2} & =\sum_{n=0}^{\infty} n !\left\|f_{N, n}\right\|_{L^{2}\left([a ; b]^{n} ; \mathscr{L}_{2}^{n}\left(\mathbb{\boxplus}^{\times n} ; H\right)\right)}^{2} \\
& =\sum_{n=0}^{\infty}\left\|\psi_{N, n}\right\|_{L^{2}\left(S_{n}^{-N, N} ; \mathscr{L}_{2}^{n}\left(\mathbb{\boxplus}^{\times n} ; H\right)\right)}^{2} .
\end{aligned}
$$

Suppose that the functions $f_{N, n}$ and $\psi_{N, n}$ are defined everywhere in $\mathbb{R}^{n}$ and are equal to zero on $\mathbb{R}^{n} \backslash[-N, N]^{n}$ and $\mathbb{R}^{n} \backslash S_{n}^{-N^{\prime}, N^{\prime},}$ correspondingly.

It follows from the equality $E\left[F_{N^{\prime \prime}} \mid \mathscr{B}_{N^{\prime}}^{-N^{\prime}}\right]=F_{N^{\prime}}$ where $N^{\prime \prime}>N^{\prime}$, from the uniqueness of the representation (89), and from the assertion of Lemma 4 that the equality $\psi_{N^{\prime}, n}=$ $\psi_{N^{\prime \prime}, n} \cdot 1_{S_{n}^{-N^{\prime}, N^{\prime}}}$ holds true and therefore $f_{N^{\prime}, n}=f_{N^{\prime \prime}, n} \cdot 1_{\left[-N^{\prime}, N^{\prime}\right]^{n}}$ for $N^{\prime \prime}>N^{\prime}$ and any $n \in \mathbb{N}$. It follows from here that for any $n \in \mathbb{N}$ there exists a unique function $\psi_{n}$ such that for any $N \in \mathbb{N}$ the equality $\psi_{n}=\psi_{N, n} \cdot 1_{S_{n}^{-N^{\prime}, N^{\prime}}}$ holds true and there exists a unique function $f_{n}$ such that for any $N \in \mathbb{N}$ it holds $f_{n}=f_{N, n} \cdot 1_{\left[-N^{\prime}, N^{\prime}\right]^{n}}$. By the estimates

$$
\begin{aligned}
& \left\|\psi_{N, n}\right\|_{L^{2}\left(\mathbb{R}^{n} ; \mathscr{L}_{2}^{n}\left(\mathbb{M}^{\times n} ; H\right)\right)}^{2} \\
& =\left\|\psi_{N, n}\right\|_{L^{2}\left(S_{n}^{-N, N} ; \mathscr{L}_{2}^{n}\left(\mathbb{M}^{\times n} ; H\right)\right)}^{2} \leq\left\|F_{N}\right\|_{L^{2}(\Omega ; H)}^{2}, \\
& \left\|f_{N, n}\right\|_{L^{2}\left(\mathbb{R}^{n} ; \mathscr{L}_{2}^{n}\left(\mathbb{\boxplus}^{\times n} ; H\right)\right)}^{2} \\
& \quad=\left\|f_{N, n}\right\|_{L^{2}\left([-N, N]^{n} ; \mathscr{L}_{2}^{n}\left(\mathbb{M}^{\times n} ; H\right)\right)}^{2} \leq\left\|F_{N}\right\|_{L^{2}(\Omega ; H)}^{2},
\end{aligned}
$$

it follows that

$$
\psi_{n}:=\lim _{N \rightarrow \infty} \psi_{N, n}, \quad f_{n}:=\lim _{N \rightarrow \infty} f_{N, n},
$$


in the space $L^{2}\left(\mathbb{R}^{n} ; \mathscr{L}_{2}^{n}\left(\mathbb{\boxplus}^{\times n} ; H\right)\right)$. Consequently,

$$
J_{n}\left(\psi_{n}\right):=\lim _{N \rightarrow \infty} J_{n}\left(\psi_{N, n}\right), \quad I_{n}\left(f_{n}\right)=\lim _{N \rightarrow \infty} I_{n}\left(f_{N, n}\right)
$$

exist in the space $L^{2}(\Omega ; H)$. Passing to the limits in the equalities (89) and (90) we obtain (87) and (88).

\section{Conflict of Interests}

The author declares that there is no conflict of interests regarding the publication of this paper.

\section{Acknowledgments}

This work is partially supported by the Ministry of Education and Science of Russian Federation (Program 1.1016.2011), by RFBR, Project 13-01-00090, and by the Program of State Support of RF Leading Universities (Agreement no. 02.A03.21.0006 of 27.08.2013).

\section{References}

[1] K. Itô, "Multiple Wiener integral," Journal of the Mathematical Society of Japan, vol. 3, pp. 157-169, 1951.

[2] P. Malliavin, "Stochastic calculus of variation and hypoelliptic operators," in Proceedings of the International Symposium on Stochastic Differential Equations. (Research Institute For Mathematical Sciences, Kyoto University, Kyoto, 1976), pp. 195-263, John Wiley \& Sons, New York, NY, USA, 1978.

[3] G. da Prato, Introduction to Stochastic Analysis and Malliavin Calculus, vol. 6 of Appunti, Scuola Normale Superiore di Pisa, Edizioni della Normale, Pisa, Italy, 2007.

[4] P. Malliavin and A. Thalmaier, Stochastic Calculus of Variations in Mathematical Finance, Springer, Berlin, Germany, 2006.

[5] G. di Nunno, B. Øksendal, and F. Proske, Mallivin Calculus for Levy Processeswith Applications to Finance, Springer, Berlin, Germany, 2009.

[6] G. DaPrato and J. Zabczyk, Stochastic Equations in Infinite Dimensions, Cambridge University Press, New York, NY, USA, 1992.

[7] I. V. Melnikova and M. A. Alshanskiy, "The generalized wellposedness of the Cauchy problem for an abstract stochastic equation with multiplicative noise," Proceedings of the Steklov Institute of Mathematics, vol. 280, supplement 1, pp. 134-150, 2013.

[8] D. A. Filipović, Consistency Problems for Heath-Jarrow-Morton Interestrate Models, vol. 1760 of Lecture notes in Mathematics, Springer, 2001.

[9] I. Ekeland and E. Taflin, "A theory of bond portfolios," Annals of Applied Probability, vol. 15, no. 2, pp. 1260-1305, 2005.

[10] B. Øksendal, Stochastic Differential Equations. An Introduction With Applications, Springer, New-York, NY, USA, 2010. 


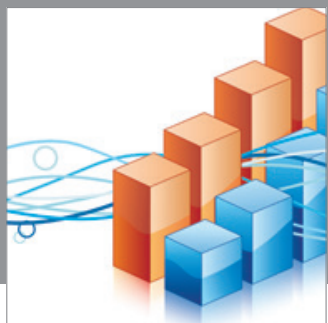

Advances in

Operations Research

mansans

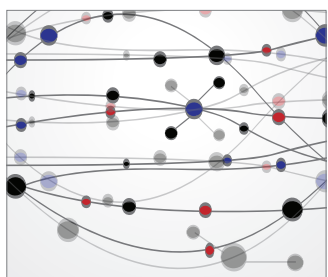

The Scientific World Journal
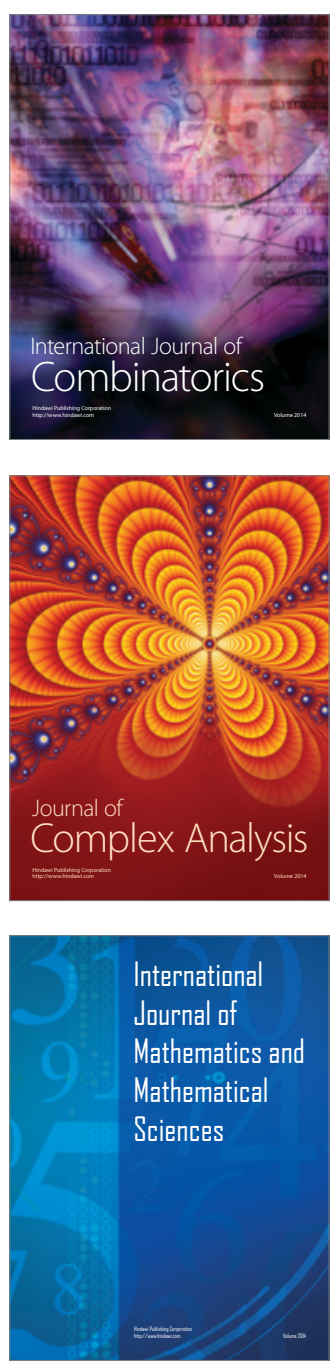
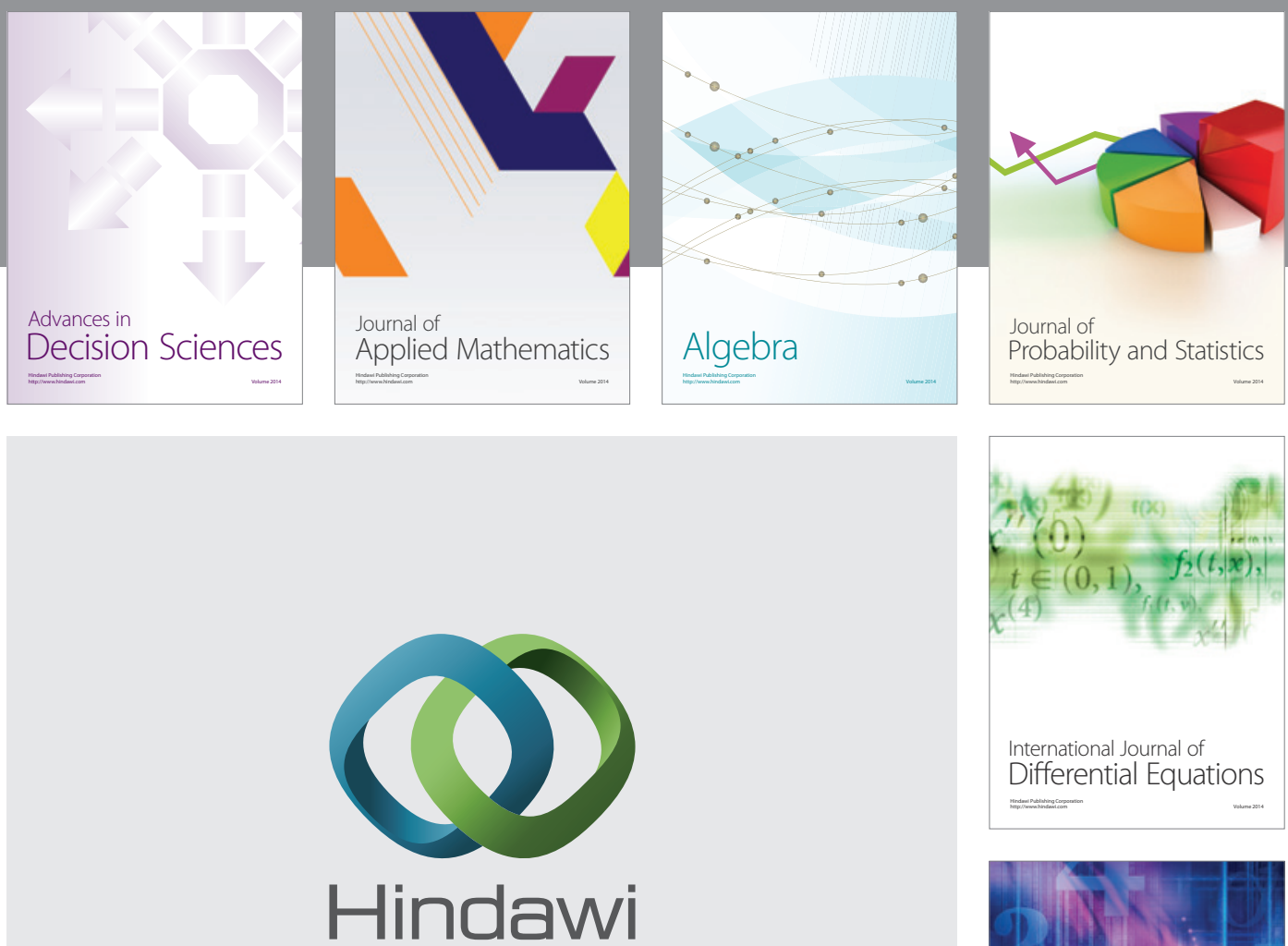

Submit your manuscripts at http://www.hindawi.com
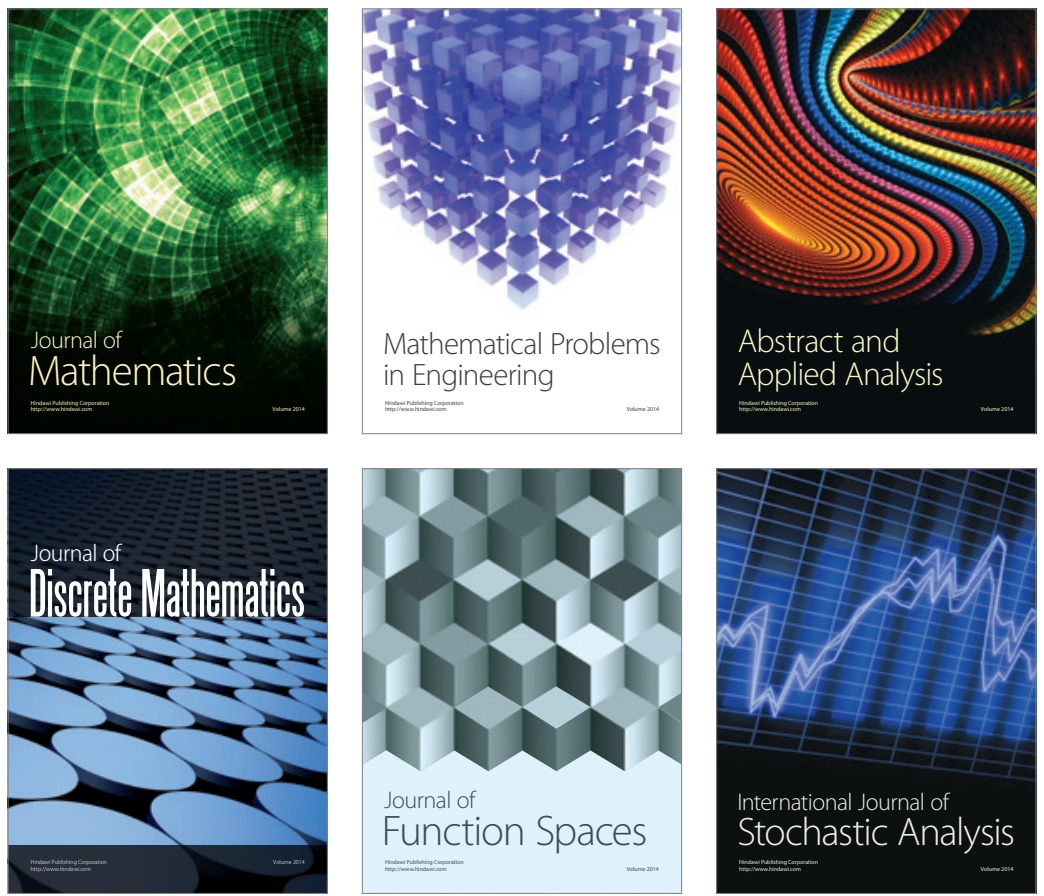

Journal of

Function Spaces

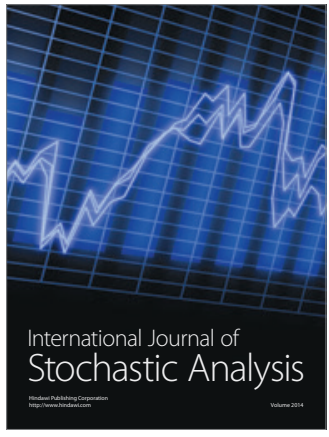

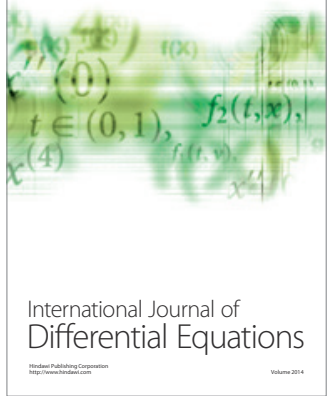
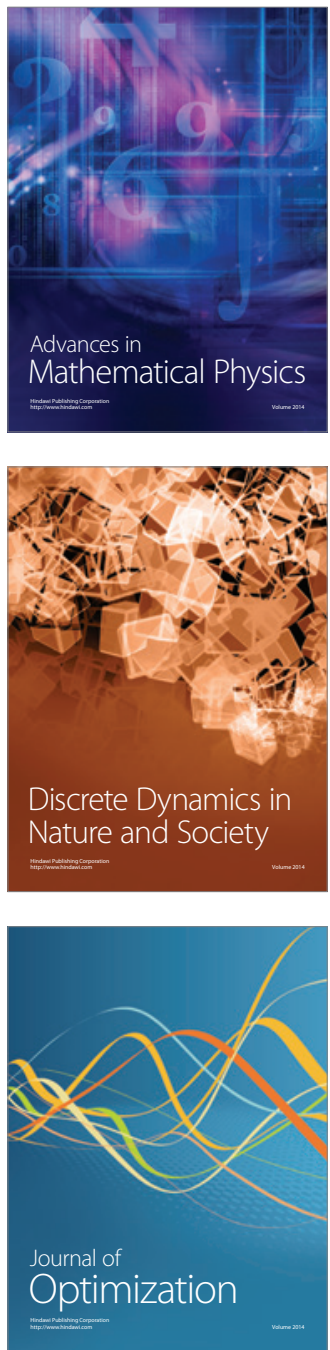